\title{
A Survey of LIDAR Technology and its Use in Spacecraft Relative Navigation
}

\author{
John A. Christian ${ }^{*}$ \\ West Virginia University, Morgantown, WV 26506 \\ Scott Cryan ${ }^{\dagger}$ \\ NASA Johnson Space Center, Houston, TX 77058
}

\begin{abstract}
This paper provides a survey of modern LIght Detection And Ranging (LIDAR) sensors from a perspective of how they can be used for spacecraft relative navigation. In addition to LIDAR technology commonly used in space applications today (e.g. scanning, flash), this paper reviews emerging LIDAR technologies gaining traction in other non-aerospace fields. The discussion will include an overview of sensor operating principles and specific pros/cons for each type of LIDAR. This paper provides a comprehensive review of LIDAR technology as applied specifically to spacecraft relative navigation.
\end{abstract}

\section{Introduction}

$\mathrm{T}$ HE problem of orbital rendezvous and docking has been a consistent challenge for complex space missions since before the Gemini 8 spacecraft performed the first successful on-orbit docking of two spacecraft in 1966. Over the years, a great deal of effort has been devoted to advancing technology associated with all aspects of the rendezvous, proximity operations, and docking (RPOD) flight phase. After years of perfecting the art of crewed rendezvous with the Gemini, Apollo, and Space Shuttle programs, NASA began investigating the problem of autonomous rendezvous and docking (AR\&D) to support a host of different mission applications. Some of these applications include autonomous resupply of the International Space Station (ISS), robotic servicing/refueling of existing orbital assets, and on-orbit assembly. ${ }^{1}$ The push towards a robust AR\&D capability has led to an intensified interest in a number of different sensors capable of providing insight into the relative state of two spacecraft. The present work focuses on exploring the state-of-the-art in one of these sensors - LIght Detection And Ranging (LIDAR) sensors. It should be noted that the military community frequently uses the acronym LADAR (LAser Detection And Ranging) to refer to what this paper calls LIDARs.

A LIDAR is an active remote sensing device that is typically used in space applications to obtain the range to one or more points on a target spacecraft. As the name suggests, LIDAR sensors use light (typically a laser) to illuminate the target and measure the time it takes for the emitted signal to return to the sensor. Because the light must travel from the source, to the target object, and back to the detector, the range to the observed point may be computed (ideally) as,

$$
r=\frac{c t}{2}
$$

where $r$ is the range from the sensor to a point on the target object, $c$ is the speed of light, and $t$ is the laser time-offlight (ToF). There are various practical concerns that complicate this equation in practice, but these are not addressed here.

The remainder of this paper will focus on the different types of LIDAR sensors and how each of them obtain laser ToF measurements to various points on in the observed scene (which in turn are used to compute range). A classification of LIDAR types is presented and then the historical use of LIDARs in space systems is summarized. With all this in mind, the various pros and cons for each type of LIDAR system are described from the perspective of their use as a relative navigation sensor.

\footnotetext{
${ }^{*}$ Assistant Professor, Department of Mechanical \& Aerospace Engineering, and AIAA Senior Member.

${ }^{\dagger}$ Engineer, Aerosciences and Flight Mechanics Division.
} 


\section{A Classification of LIDAR Sensors}

LIDAR sensors may be divided into a few different categories based on how they illuminate and sense the 3D scene in front of them. Broadly speaking, we propose that most current LIDAR systems can categorized into three major groups:

1. Scanning: systems that illuminate the scene through sweeping of a "narrow" laser beam and sense the return with only a single detector

2. Detector Arrays: systems that illuminate the entire scene at once and sense the return with an array of detectors

3. Spatial Light Modulators: systems that illuminate portions of the scene in a pattern and then sense the return with a single detector (along with compressed sensing algorithms)

For the first two groups (Scanning and Detector Array) may measure the laser ToF through one of a few common mechanisms, see Table 1 . The first method is to send out discrete laser pulses/flashes and then await the return pulse at the detector. The second method is to modulate a signal onto the laser and then track the phase shift in the returning signal to measure laser ToF. The third method is to encode a pseudo-random number (PRN) sequence onto the laser and then perform an autocorrelation with the sensed return to determine laser ToF.

Table 1. A classification of LIDAR sensors.

\begin{tabular}{lccc}
\hline \hline ToF Method & Scanning & $\begin{array}{c}\text { Detector } \\
\text { Array }\end{array}$ & $\begin{array}{c}\text { Spatial Light } \\
\text { Modulator }\end{array}$ \\
\hline Pulse/Flash & $\mathrm{X}$ & $\mathrm{X}$ & \\
Continuous Wave (CW) & $\mathrm{X}$ & $\mathrm{X}$ & \\
$\begin{array}{l}\text { Pseudo-Random Number (PRN) } \\
\text { Compressed Sensing (CS) }\end{array}$ & $\mathrm{X}$ & $\mathrm{X}$ & $\mathrm{X}$ \\
\hline \hline
\end{tabular}

In the subsequent subsections, we will discuss each of thee major classes of LIDARs in more detail. Particular focus will be given to their applicability for use in spacecraft AR\&D applications. The following discussions focus on the Flash, CW, and CS methods for measuring laser ToF. For more information on PRN LIDARs, the reader is directed to Ref. [2].

\section{A. Scanning LIDARs}

The first major class of LIDARs are scanning LIDARs. These sensors use a narrow laser beam that is swept over the sensor Field of View (FOV) to obtain range measurements to objects within the scene as shown in Fig. 1. The return from this laser typically illuminates a single detector as the laser direction is changed by a set of mirrors, lenses, and/or other devices. By combining knowledge of the laser direction and the measured range, a three-dimensional (3D) point cloud of the scene may be constructed.

Scanning LIDARs have seen extensive historical use in space applications, as is discussed in Section III. Because they only use one detector (or a very small number of detectors), these sensors are relatively easy to calibrate. The user only needs to be concerned about the light sensitivity and timing for one detector. Additionally, because the LASER is typically directed by a system of lenses/mirrors, scanning LIDARs can point the narrow laser beam very precisely and create very high-resolution point clouds. Scanning LIDARs are also well suited for tracking a single object - once the laser "locks on" to the object (e.g. a reflector) it can track this object without worrying about the rest of the scene.

As their name implies, however, scanning LIDARs do contain moving parts that can wear out over time and potentially be a source of hardware failure. Further, if one desires a full 3D point cloud for an entire scene, it does take scanning LIDARs a finite amount of time to scan its entire field-of-view (FOV). The time required to compete this task varies widely amongst different systems. If the objects within the scene undergo substantial relative motion during the time required to scan the FOV there can be undesirable artifacts and/or motion blur in the resulting 3D point cloud. 


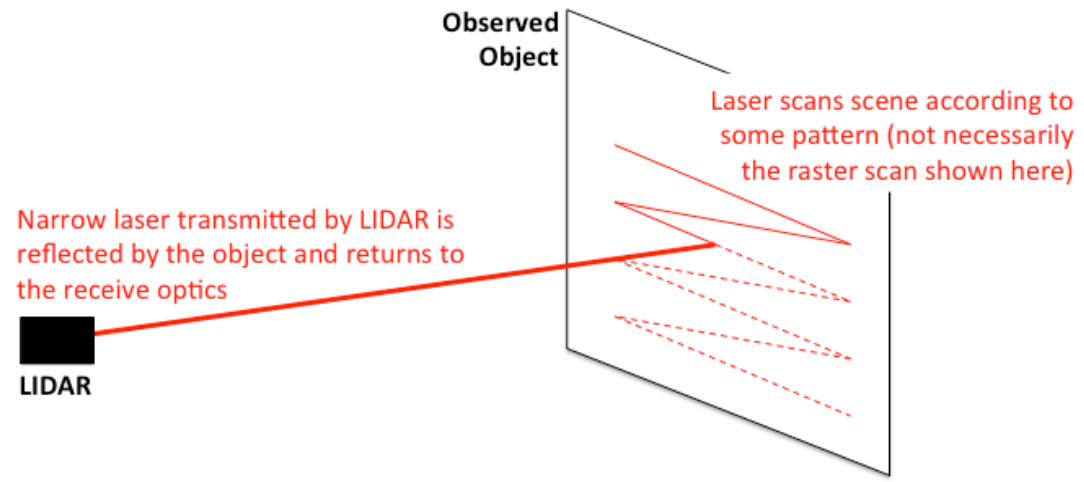

Figure 1. Notional depiction of a typical scanning LIDAR.

\section{B. Detector Array LIDARs}

The second major class of LIDARs uses flood illumination of the entire scene by a laser and individually measures the laser ToF to each pixel on a detector array. The receive optics and detector array operate in much the same way as a conventional camera. Therefore, by combining the pixel location (which maps directly to a known line-of-sight direction) with the laser ToF associated with that pixel, a 3D point cloud of the scene may be constructed. This concept is shown graphically in Fig. 2.

Within the class of LIDARs using a detector arrays, there are numerous ways that ToF can be measured at each pixel. The most common type used in space applications is the Flash LIDAR. A Flash LIDAR sends out a laser pulse (the "flash") and measures the time until the return pulse is detected at each pixel.

The second common type of LIDAR with a detector array is the Continuous Wave (CW) LIDAR (sometimes called a Time-of-Flight Camera), ${ }^{3,4}$ although it has yet to find widespread use outside of the robotics, computer vision, and home entertainment communities. CW LIDARs modulate the intensity of the laser and then measure the phase difference between the emitter and each pixel on the detector. This is done by sampling the return signal at least four times as fast as the modulated signal, as is shown in Fig. 3, and using the algorithmic approach outlined in both [3] and [4] to compute range. A typical CW LIDAR with a range of $10 \mathrm{~m}$ may be modulated at around 15 $\mathrm{MHz}$, thus requiring that the signal at each pixel be sampled at around $60 \mathrm{MHz}$. Most CW LIDARs that are commercially available today operate by modulating the laser intensity in the near infrared (NIR), making their use difficult in direct sunlight.

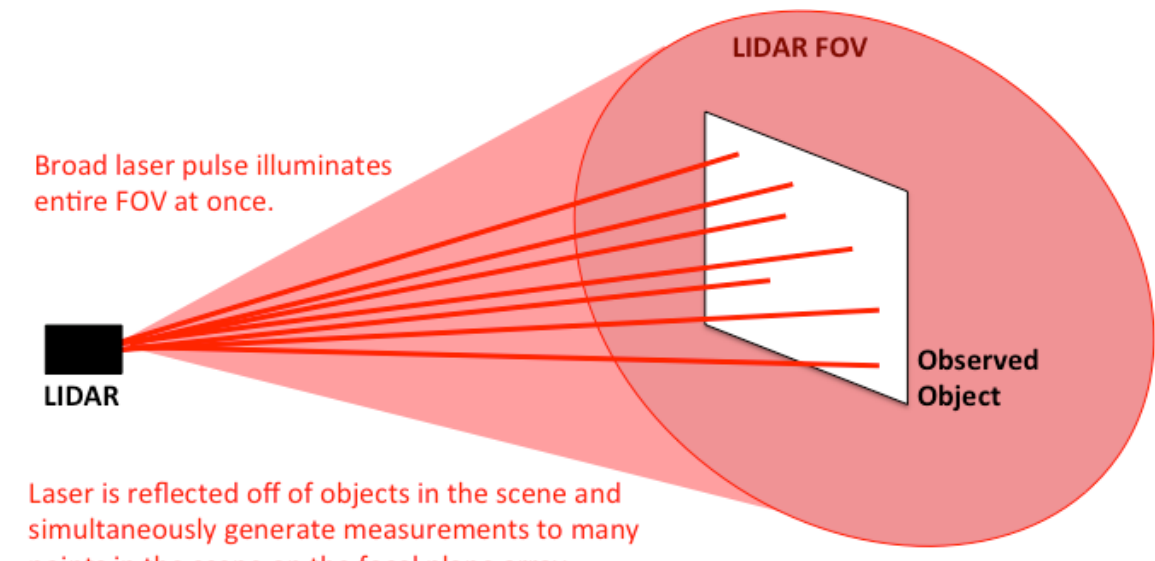

points in the scene on the focal plane array.

Figure 2. Notional depiction of detector array LIDAR. 


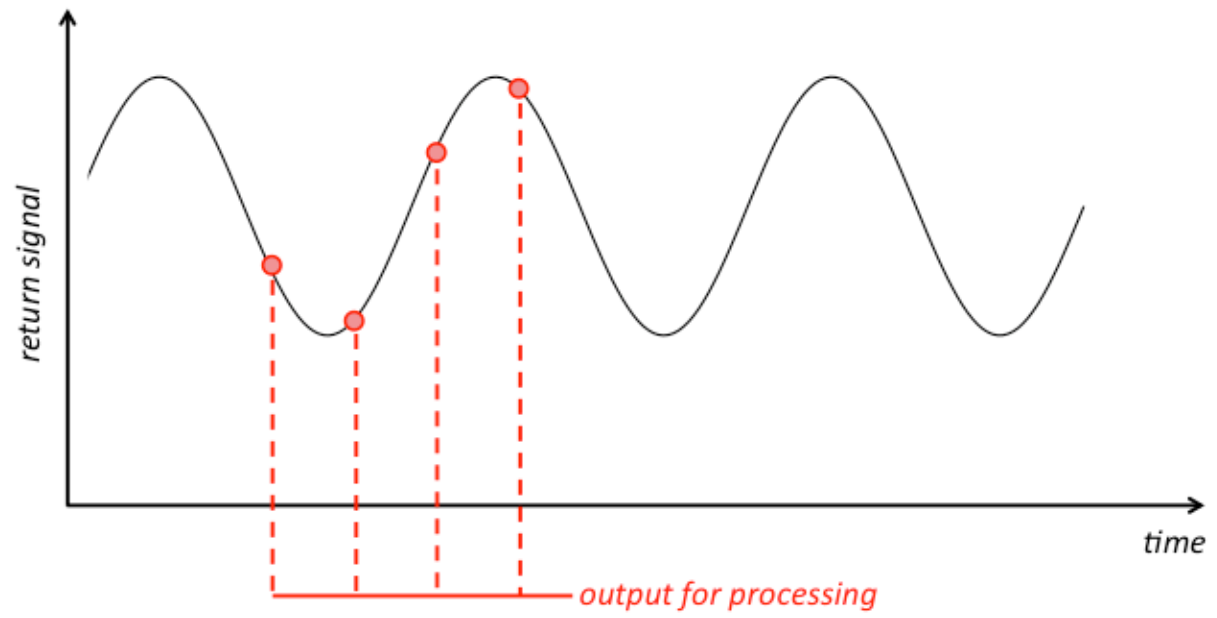

Figure 3. In CW LIDARs (ToF Cameras) phase difference is commonly computed by sampling the observed signal at four times the modulation frequency.

One of the great advantages of detector array LIDAR systems in space applications is that they have no moving parts. This removes one of the most failure-prone components of the scanning LIDAR system. These systems, however, achieve this advantage through the use of a large detector array. Because detector array LIDAR systems have more detectors, they are more challenging to calibrate. Just as each pixel in a regular camera will have it's own sensitivity to light, each pixel in these LIDAR systems must be individually calibrated for both light sensitivity and timing.

Further, there are numerous pros/cons related to the choice of Flash vs. CW for determining laser ToF. First, it is somewhat simpler to obtain accurate ToF measurements through the CW method. While similar performance can be obtained with Flash systems, it typically requires greater algorithmic complexity and more careful calibration. Second, Flash LIDARs are suitable for use at both short range and long range, while CW LIDARs commonly suffer from the phase integer ambiguity problem. Many CW LIDAR systems address this by limiting sensor performance to only close-range where the phase shift is less than half a cycle. That is,

$$
r_{\max , C W}=\frac{c}{2 f_{\text {mod }}}
$$

where $f_{\text {mod }}$ is the modulation frequency. For example, a CW system with $15 \mathrm{MHz}$ modulation frequency would have a maximum range of about $10 \mathrm{~m}$ before phase wrapping becomes a problem. The ability of Flash LIDARs to handle ranges from docking to many kilometers is one of the reasons they are currently preferred over CW systems for AR\&D applications.

The third issue to consider in choosing a method to measure ToF is range/image artifacts introduced by object motion. Flash LIDARs have the additional advantage that because the entire scene is illuminated at once with a laser pulse, the 3D point cloud for the scene is generated at once. Assuming that all relative motion in the scene is substantially slower than the speed of light, no noticeable motion blur is observed in individual Flash LIDAR images. This is not necessarily the case with CW LIDARs because of how the modulated signal is sampled to compute range. If an object moves out of a pixel during a sequence of samples, some of the samples will correspond to a return from the object (while it is still in the line-of-sight of that pixel) and the remainder of the samples will correspond to a return from whatever is behind the object (because the object has moved out of the line-of-sight of that pixel). Simple CW processing algorithms do not account for such discontinuities and inconsistencies, and the result is edge artifacts sometime called flying pixels. ${ }^{4}$

\section{Spatial Light Modulator LIDARs}

The third major class of LIDARs are those that use Spatial Light Modulators (SLMs) to illuminate the scene and then use techniques from compressed sensing (CS) to reconstruct a 3D point cloud. ${ }^{5}$ This new class of LIDAR sensor, still under development at MIT, uses neither a scanning laser (as in the first class) nor an array of detectors (as in the second class). Instead, in this third class of LIDARs, the SLM is used to sequentially illuminate subsets of 
the scene with a sequence of known patterns. Then, the time history of the laser return from the entire scene (for each of the illumination patterns) is measured by a single detector. Now, by combining some assumptions on scene geometry (e.g. piecewise planar) with CS algorithms, the time history of the returns from a set of illumination patterns may be used to reconstruct an approximation of the 3D scene.

While this type of LIDAR system has no moving parts and only a single detector, it must make approximations of the scene geometry in order to apply the CS algorithms. Further, this type of LIDAR system is still under development and is likely years away from practical application in the space environment.

\section{Review of Historical LIDAR Use for Spacecraft Relative Navigation}

\section{A. LIDARs flown on Previous Spacecraft}

\section{Trajectory Control Sensor (TCS) on the Space Shuttle}

The Trajectory Control Sensor (TCS) is a scanning LIDAR that flew on the Space Shuttle and was successfully used as a relative navigation aid during rendezvous with the Mir Space Station, the Hubble Space Telescope (HST), and the ISS. ${ }^{6}$ The TCS tracked reflectors mounted at known locations on the target object and provided the navigation system with range and bearing to the reflector being tracked. TCS first flew on STS-71 in 1995 to Mir (the Russian space station), and the last TCS flight was on STS-135 to the ISS. TCS was used on all flights to Mir (8 flights) and to the ISS. Of particular note is that use of TCS was instrumental in the development of the Rendezvous, Proximity Operations Program (RPOP). ${ }^{7}$ RPOP resided on an IBM ThinkPad laptop, and was used by the crew to "fly" the Space Shuttle while performing rendezvous and proximity operations with another vehicle.

\section{Videometer (VDM) on the ESA Automated Transfer Vehicle (ATV)}

The Videometer (VDM), manufactured by Sodern (now EADS - Sodern), is a relative navigation sensor used by ESA's ATV. The VDM has flown on all of the ESA ATV missions to date (ATV-1 through ATV-4), and is planned for use on ATV-5. The VDMs provide range, range-rate and line-of-sight measurements to the ATV GNC algorithms from 300 meters to docking at rate of $1 \mathrm{~Hz}$. The VDM can also provide relative position and relative attitude in support of docking from 30 meters to docking. The sensor is based on Sodern's SED16 star tracker. The VDMs make use of retro-reflector assemblies mounted to the ISS Service Module (SM) aft end. There are three multi-reflector planar assemblies (consisting of seven retro-reflectors in a circular, planar pattern) arranged in a triangular pattern. Additionally, there is a pyramidal array of five retro-reflectors (four in a flat plane, one in the center that is $10 \mathrm{~cm}$ above the planar array). The VDM utilizes all of the retro-reflector assemblies at long to midranges. The VDM relies on the pyramidal array to compute the relative position and relative attitude solution when the range is under 30 meters. The VDM operates similarly to a Flash LIDAR, whereby laser diodes surrounding the detector aperture, strobe the target vehicle and the return light/energy hits the detector.

\section{RendezVous Sensor (RVS) / Telegoniometer (TGM) on HTV / ATV}

The RVS/TGM is manufactured by Jena Optronik GmbH (now a subsidiary of Astrium-EADS). The RVS was developed and tested on two STS DTOs (STS-84 and STS-86), ${ }^{8}$ and is now the primary relative navigation sensor on JAXA's H-II Transfer Vehicle (HTV) resupply spacecraft. Under the name TGM (ESA designation) the same fundamental sensor is used for fault detection, isolation, and recovery (FDIR) on ESA's ATV. The RVS and TGM are essentially the same sensor, with slightly different operating modes and target pattern recognition schemes encoded internally. The RVS/TGM has flown on all of the HTV and ATV flights to date without any issues. The RVS can operate from $1.5 \mathrm{~km}$ to docking. The RVS is a scanning LIDAR, employing two scanning mirrors. Use of retro-reflectors is required by RVS on both ATV and HTV. For HTV, the RVS would acquire the Japanese Experiment Module (JEM) mounted hemispherical retro-reflector assemblies while on the RBAR, at a range of approximately 750 meters. The RVS would then provide range, range-rate and line of sight angles from HTV to the reflectors from acquisition until the HTV reached the "berthing box", would hold in the berthing box while under RVS control until the HTV was grappled by the ISS robotic arm.

\section{B. LIDAR Flight Experiments for New Sensors}

Between 2009 and 2011 there were a total of six flight experiments, or Development Test Objectives (DTOs), flown on Space Shuttle missions to the ISS to test a new generation of LIDAR technology. These six tests consisted of three flights of the Neptec TriDAR (STS-128, STS-131, and STS-135), ${ }^{9}$ two flights of the ASC DragonEye Flash 
LIDAR (STS-127 and STS-133), ${ }^{10,11}$ and one flight of the Ball Aerospace Technology Corp. (BATC) Vision Navigation Sensor (VNS) Flash LIDAR (STS-134). ${ }^{12}$ The sensors were mounted in the spare TCS slot on the Orbiter Docking System (ODS) truss, adjacent to the Orbiter's operating TCS. Each supplier performed data analyses of their sensor's performance to further their understanding as well as determine the best possible paths for sensor refinement and advancement. The NASA Engineering and Safety Center (NESC) funded an independent analysis task that looked at each sensors' performance and compared the results against each sensors' specifications. ${ }^{13}$

\section{Neptec's TriDAR}

The TriDAR had flown on three (3) flights (STS-128, STS-131 and STS-135). TriDAR is a scanning LIDAR that does that utilizes the multiple returns to generate a point-cloud. The point-cloud is then processed to generate a range and bearing solution at long ranges. At shorter ranges, the TriDAR operates in a triangulation mode employing a different detector and scanning mirrors. In triangulation mode, the point-cloud is processed to generate a relative position and relative attitude by matching the point-cloud shape to the target vehicle's model within the sensor.

The TriDAR underwent updates between each flight, enhancing the sensor's performance at each step of the evolution. The changes included increasing structural rigidity and firmware/software updates to the measurement processing. The sensor performance showed improvements with each flight. STS-128 was the first space flight of the TriDAR. The results from STS-128 and STS- $131^{14}$ led to advancements in improvement for STS- 135 .

\section{ASC's DragonEye}

The ASC DragonEye was selected by SpaceX as their primary means for performing proximity operations and capture. Prior to flying on their Dragon vehicle, SpaceX performed two flight experiments (DTOs) on STS-127 and STS-133. The DragonEye is a Flash LIDAR, employing a InGaAs Avalanche Photo-Diode detector array, comprised of 128x128 pixels (16,384 total pixels). The DragonEye operated at $5 \mathrm{~Hz}$, but can operate at faster rates. Based on laser power and receive optics, the operational range for DragonEye was under 1,000 meters. For Dragon vehicle application, maximum operational range did not need to exceed 1,000 $\mathrm{m}$, as transition from relative GPS (RGPS) to DragonEye was planned to occur around $750 \mathrm{~m}$ from ISS.

During STS-127, ${ }^{10,11}$ the DragonEye captured data at a range from $762 \mathrm{~m}$ all the way through to docking. Raw data analysis showed that a target-less solution (not utilizing retro-reflectors) could be achieved with the large number of data points in each frame. DragonEye performance during the approach and docking demonstrated that flash LIDARs can be used in rendezvous and docking.

The STS-133 flight applied lessons learned from the 127 flight, ground testing and SBIR research. Advancements were made to the laser assembly, and different detector settings selected. The settings selected for this flight were to improve the long-range performance of the DragonEye, sacrificing the short-range performance. The objective was to determine if the longer-range performance would help the Dragon vehicle when it would perform the demonstration mission not long after the DTO was to be completed. The data collected on both flights were instrumental to SpaceX in determining their sensor settings for the Dragon flights to ISS.

\section{Ball's Vision Navigation Sensor (VNS)}

The BATC VNS Flash LIDAR was flown on STS-134 as part of the Sensor Test for Orion RelNav Risk Mitigation (STORRM) DTO. ${ }^{12,15}$ The VNS is currently planned to be the primary relative navigation sensor for the Orion Multi-Purpose Crew Vehicle (MPCV). The VNS has a 256x256 focal plane array (65,536 total pixels) and a variable field of illumination that can be set to either 12 deg or 20 deg. The narrow field of illumination is typically used at long range, while the wide field of illumination is typically used at short range. The receive optics FOV, however, is fixed to 20 deg; thus, the flash will fill varying amounts of the FOV depending on the selected field of illumination. During the STORRM DTO Flash LIDAR images were collected at a rate of $30 \mathrm{~Hz}$ and the sensor demonstrated its capability of measuring range from over $5 \mathrm{~km}$ through docking.

\section{Conclusions}

LIDARs are an important sensor technology for enabling autonomous rendezvous and docking in future spaceflight missions. This paper has reviewed the common classes of LIDARs available today and has discussed some of their pros/cons with respect to relative navigation. This overview was followed by a review of previously flow LIDAR systems and a discussion of new sensors that are under development for future vehicles. Although other LIDAR 
technologies exist, variations on scanning LIDARs and Flash LIDARs are expected to be the dominant type of LIDAR technology used in space applications for the foreseeable future.

\section{References}

${ }^{1}$ Naasz, B., and Moreau, M., “Autonomous RPOD Challenges for the Coming Decade,” $35^{\text {th }}$ Annual AAS Guidance \& Control Conference, Breckenridge, CO, 3-8 February 2012.

${ }^{2}$ Takeuchi, N., Sugimoto, N., Baba, H., and Sakurai, K., “Random Modulation CW LIDAR,” Applied Optics, Vol. 22, No. 9, May 1983, pp 1382-1386.

${ }^{3}$ Foix, S., Alenya, G., and Torras, C., “Lock-in Time-of-Flight (ToF) Cameras: A Survey,” IEEE Sensors Journal, Vol. 11, No. 9, September 2011, pp 1917-1926.

${ }^{4}$ Dal Mutto, C., Zanuttigh, P., and Cortelazzo, G., Time-of-Flight Cameras and Microsoft Kinect, Springer, New York, NY, 2012.

${ }^{5}$ Kirmani, A., Colaco, A., Wong, F., and Goyal, V., "Exploiting Sparsity in Time-of-Flight Range Acquisition Using a Single Time-Resolved Sensor,” Optical Express, Vol. 19, No. 22, October 2011, pp 21485-21507.

${ }^{6}$ Goodman, J., "History of Space Shuttle Rendezvous and Proximity Operations,” Journal of Spacecraft and Rockets, Vol. 43, No. 5, September-October 2006, pp 944-959.

${ }^{7}$ Clark, F., Spehar, P., Brazzel, J., and Hinkel, H., "Laser-Based Relative Navigation and Guidance for Space Shuttle Proximity Operations,” $26^{\text {th }}$ Annual AAS Guidance \& Control Conference, Breckenridge, CO, 5-9 February 2003.

${ }^{8}$ Moebius, B., and Kolk, K.-H., "RendezVous Sensor for Automatic Guidance of Transfer Vehicles to ISS Concept of the Operational Modes Depending on Actual Optical and Geometrical-Dynamical Conditions,” Photonics for Space Environments VII, Proceedings of SPIE, Vol. 4134, 2000.

${ }^{9}$ Kearns, M., TriDAR: Test Results From Three Space Shuttle Missions, Neptec Design Group, Ltd., 2012.

${ }^{10}$ NASA, "STS-127, A Porch in Space”, STS-127 press kit, June 2009.

${ }^{11}$ Wooster, P., “DragonEye DTO 701B LIDAR Report”, Space Exploration Technologies, 11 June 2009.

${ }^{12}$ Christian, J., Hinkel, H., D’Souza, C., Maguire, S., and Patangan, M., “The Sensor Test for Orion RelNav Risk Mitigation (STORRM) Development Test Objective,” AIAA Guidance, Navigation, and Control Conference, Portland, OR, 8-11 August 2011.

${ }^{13}$ NESC GNC TDT (Dennehy, Parker, Cryan, Clark, Christian, Valdez, Heaton, Patangan, Spiers, Brown), "Relative Navigation Light Detection and Ranging (LIDAR) Sensor Development Test Objective (DTO) Performance Verification”, NESC T1-11-00753, 11 April 2013.

${ }^{14}$ Ruel, S., Luu, T. and Berube, A., "Space Shuttle Testing of the TriDAR 3D Rendezvous and Docking Sensor”, Journal of Field Roboitcs, Vol. 29, No. 4, 2012 pp 535-553.

${ }^{15}$ Gravseth, I., Rohrschneider, R., and Masciarelli, J., "Vision Navigation Sensor (VNS) Results from the STORRM Mission,” 35th Annual AAS Guidance and Control Conference, Breckenridge, CO, 3-8 Feb 2012. 